\title{
Recent Developments and Applications on Discrete Fractional Equations and Related Topics
}

\author{
Shurong Sun, ${ }^{1}$ Thabet Abdeljawad, ${ }^{2,3}$ and Jehad Alzabut ${ }^{2}$ \\ ${ }^{1}$ School of Mathematical Sciences, University of Jinan, Jinan, Shandong 250022, China \\ ${ }^{2}$ Department of Mathematics and Physical Sciences, Prince Sultan University, P.O. Box 66833, Riyadh 11586, Saudi Arabia \\ ${ }^{3}$ Department of Mathematics and Computer Science, Cankaya University, 06530 Ankara, Turkey
}

Correspondence should be addressed to Shurong Sun; sshrong@163.com

Received 30 October 2013; Accepted 30 October 2013

Copyright (C) 2013 Shurong Sun et al. This is an open access article distributed under the Creative Commons Attribution License, which permits unrestricted use, distribution, and reproduction in any medium, provided the original work is properly cited.

Fractional differential equations have been of great interest amongst researchers over the last two decades. Due to the widespread applications in science and technology, research within these types of equations has led to new developments in the theory of fractional difference equations as well as fractional $q$-difference equations. On the other hand, the study of dynamic equations on time scales has been rapidly growing. Indeed, the theory of dynamic equations unifies those of differential and difference equations. Therefore, to establish a general definition for the fractional derivative on an arbitrary time scale, the unification of the theories of fractional differential equations and discrete fractional equations has become interesting and useful.

This issue on recent developments and applications on discrete fractional equations and related topics aims to provide better understanding for fractional differential equations, fractional difference equations, fractional $q$-difference equations, and their basic theories and applications. This issue contains 15 fascinating papers of different disciplines, and most of them were focusing on fractional differential equations, fractional difference equations, and dynamic equations on time scale.

There are many different approaches to obtain fractional derivatives. T. Abdeljawad et al. gave the definitions of the delta left and nabla right fractional differences and sums with binomial coefficients. Indeed, they used the discrete version of the $Q$-operator and some discrete fractional dual identities to prove that the presented fractional differences and sums coincide with the discrete Riemann ones by the method of iterating the integral. T. Abdeljawad investigated two types of dual identities for Caputo fractional differences and the relation between Riemann and Caputo fractional differences. He showed that the delta and nabla discrete MittagLeffler functions are confirmed by solving Caputo type linear fractional difference equations. Nevertheless, R. W. Ibrahim and H. A. Jalab extended the complex step method by employing the fractional calculus differential operator, derived several approximations for computing the fractional order derivatives, and studied the stability of the generalized fractional complex step approximations for an analytic test function.

The qualitative theory including the existence and uniqueness of solutions, stability, asymptotic, oscillation, and periodicity is a crucial subject in the study of differential equations. F. Chen and Y. Zhou discussed the existence of solutions for antiperiodic boundary value problem and the Ulam stability for nonlinear fractional difference equations. Z. Han et al. investigated the oscillation for a class of fractional differential equations. However, B. Wu studied a class of pseudodifferential equations involving $p$-adic fractional pseudodifferential operator. The fundamental solution of the equation was obtained. Moreover, the continuity and uniqueness of solution which belong to the Sobolev type space were obtained under certain conditions. W. Lvis concerned with the existence and uniqueness for a discrete fractional $p$ Laplacian boundary value problem. However, C. Tunç and M. Ates considered the boundedness of solutions to differential equations of fourth order with oscillatory restoring and forcing terms. P. Zhao and H. Man employed the characteristics-mixed method for approximating the solution to 
a convection-dominated transport problem. K. Li and C. Zhang studied the solutions of second-order linear matrix equations on time scales, established necessary and sufficient conditions for the existence of a solution of characteristic equation, and applied two diverse solutions of characteristic equation to express the general solution of the matrix equations on time scales. $\mathrm{H}$. Wu and Y. Zhou studied a planthare model subjected by the effect of impulses and gave some sufficient conditions for the existence of at least one positive periodic solution. J. Zhang et al. established some sufficient conditions for the existence and uniqueness of positive solutions to a class of initial value problems for impulsive fractional differential equations involving the Caputo fractional derivative by a fixed point theorem for mixed monotone operators.

The fractional differential equations play an important role in various fields of science and engineering. With the help of fractional calculus, it has been found that many natural phenomena can be described using certain mathematical models. Indeed, H. A. Jalab and R. W. Ibrahim introduced a texture enhancement technique for medical images by using fractional differential masks based on Srivastava-Owa fractional operators. A 2D isotropic gradient mask based on generalized fractional operators is constructed. Texture enhancement performance was measured by applying experiments according to visual perception and by using Sobel/Canny edge filters and gray level cooccurrence matrix. They also discussed the capability of the fractional differential mask for texture enhancement. The experiments and analysis showed that the operator can extract subtle information and make the edges prominent. H. A. Jalab and R. W. Ibrahim introduced a system of fractional order derivative for a uniformly sampled polynomial signal by employing the generalized fractional differential operator and discussed the convergence of the system to a fractional time differentiator.

In conclusion, we hope that the papers published in this issue will enrich the readers' knowledge and stimulate researchers to extend, generalize, and apply the established results.

\section{Acknowledgment}

We would like to thank the editorial board members of this journal for their support and help throughout the preparation of this special issue.

Shurong Sun

Thabet Abdeljawad Jehad Alzabut 


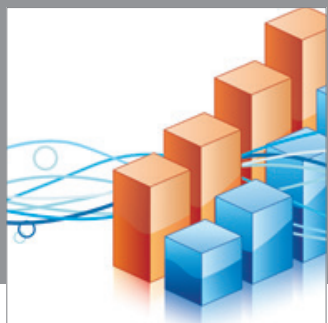

Advances in

Operations Research

mansans

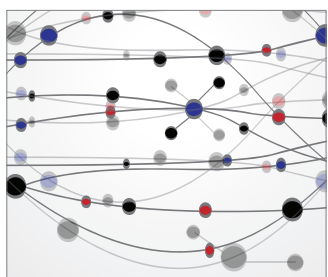

The Scientific World Journal
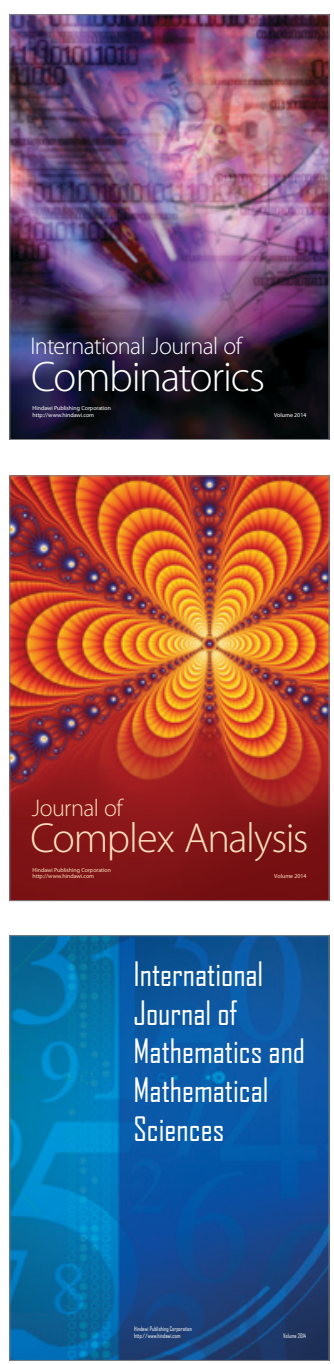
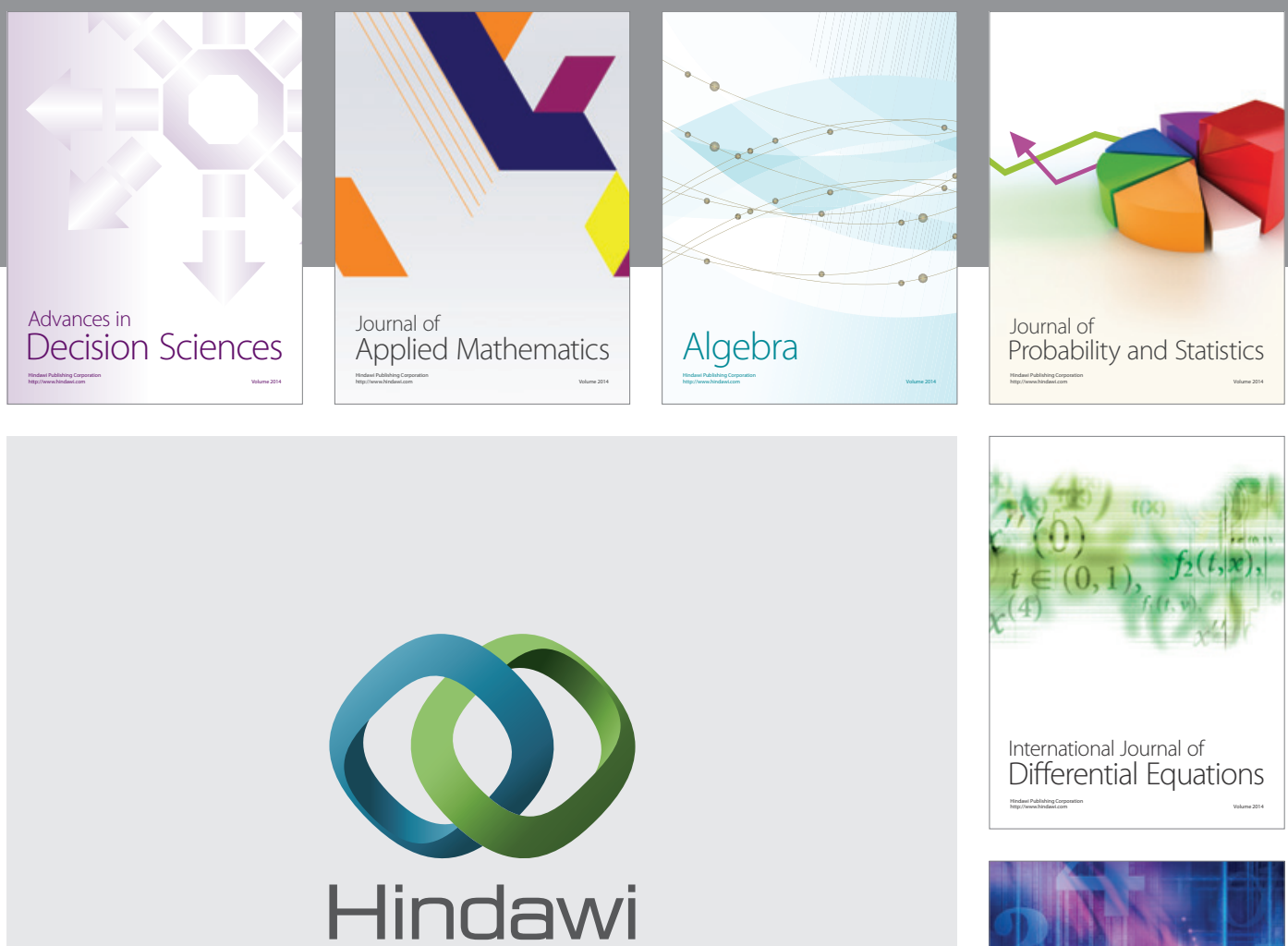

Submit your manuscripts at http://www.hindawi.com
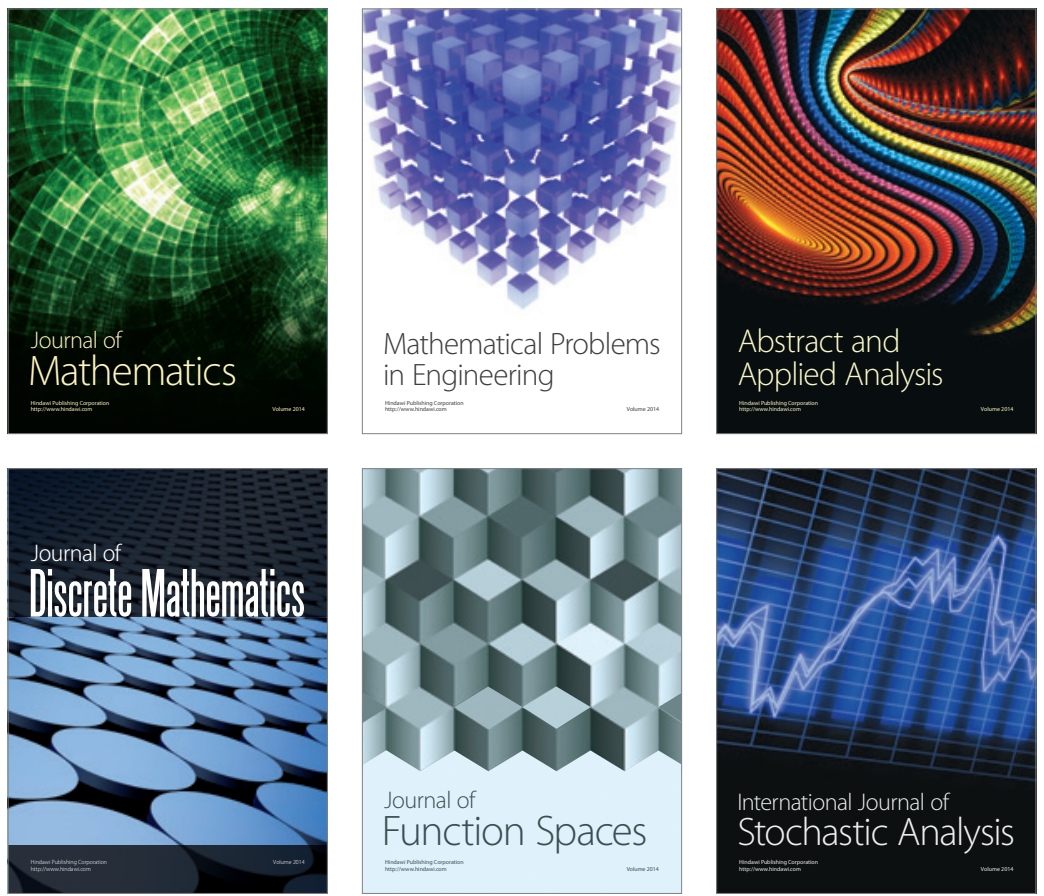

Journal of

Function Spaces

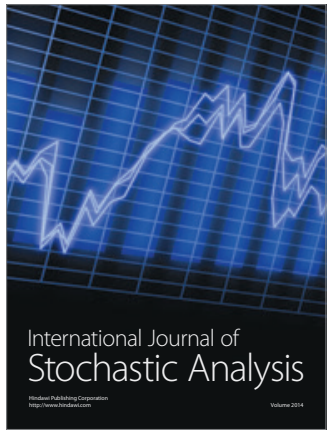

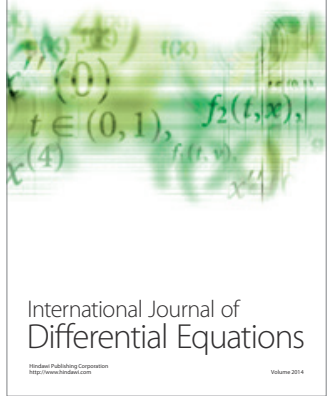
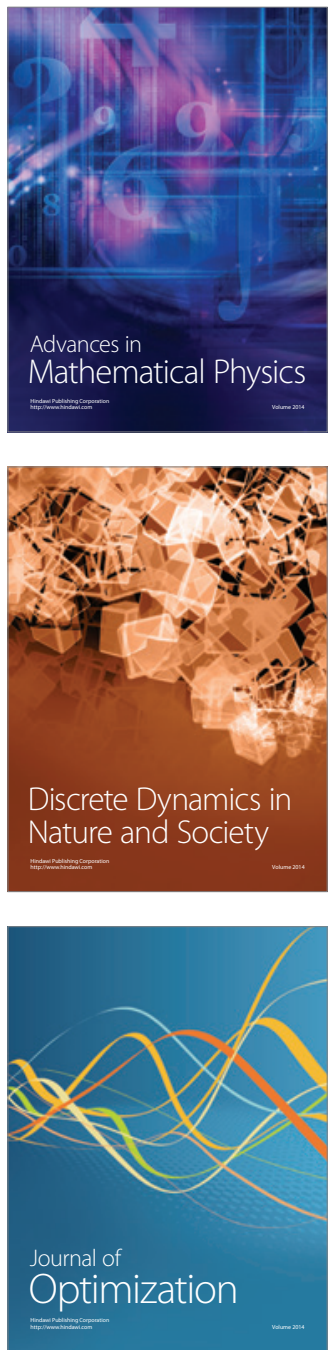